NISTIR 7901

\title{
Best Practices and Performance Metrics Using Force Control for Robotic Assembly
}

Jeremy Marvel

Joe Falco

http://dx.doi.org/10.6028/NIST.IR.7901

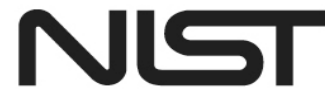

National Institute of Standards and Technology U.S. Department of Commerce 


\title{
Best Practices and Performance Metrics Using Force Control for Robotic Assembly
}

\author{
Jeremy Marvel \\ Joe Falco \\ Intelligent Systems Division \\ Engineering Laboratory
}

http://dx.doi.org/10.6028/NIST.IR.7901

November 2012

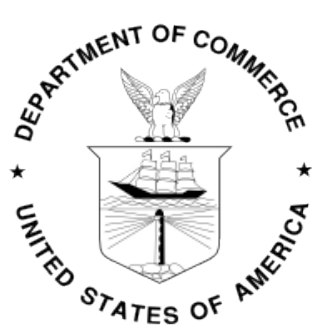

U.S. Department of Commerce

Rebecca Blank, Acting Secretary

National Institute of Standards and Technology Patrick D. Gallagher, Under Secretary of Commerce for Standards and Technology and Director 


\section{Best Practices and Performance Metrics Using Force Control for Robotic Assembly}

Jeremy Marvel, Joe Falco

\begin{tabular}{|c|c|}
\hline \multicolumn{2}{|r|}{$\begin{array}{c}\text { Table of Contents } \\
\text { DISCLAIMER }\end{array}$} \\
\hline \multicolumn{2}{|r|}{$\begin{array}{l}\text { DISCLAIMER } \\
\text { I. }\end{array}$} \\
\hline \multicolumn{2}{|r|}{ II. Robotics in Assembly } \\
\hline \multicolumn{2}{|r|}{$\begin{array}{ll}\text { II. } & \text { Robotics in Assembly } \\
\text { III. } & \text { Assembly and Robot Dexterity }\end{array}$} \\
\hline \multirow{2}{*}{$\begin{array}{l}\text { IV. } \\
\text { V. }\end{array}$} & 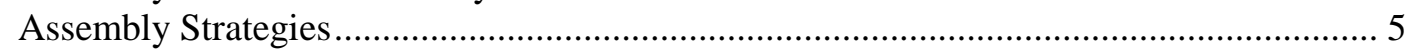 \\
\hline & Force Control ............. \\
\hline A. & Explicit Force Control. \\
\hline B. & Stiffness Control ............................. \\
\hline C. & Impedance Control... \\
\hline D. & Admittance / Accommodation Control \\
\hline \multirow{2}{*}{$\begin{array}{l}\text { E. } \\
\text { F. }\end{array}$} & Implicit Force Control ... \\
\hline & Natural Admittance Control...... \\
\hline VI. & Commercial Robot Solutions ................ \\
\hline \multirow{2}{*}{ VII } & Metrics for Assessing Force Control .... \\
\hline & 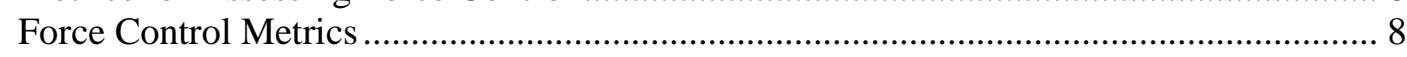 \\
\hline \multirow{2}{*}{$\mathrm{VII}$} & 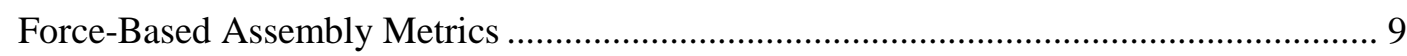 \\
\hline & 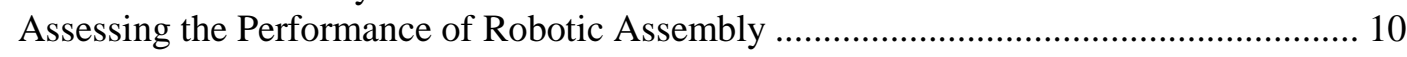 \\
\hline & 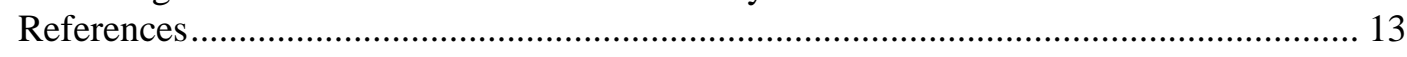 \\
\hline
\end{tabular}




\section{DISCLAIMER}

Certain trade names and company products are mentioned in the text or identified in certain illustrations. In no case does such an identification imply recommendation or endorsement by the National Institute of Standards and Technology, nor does it imply that the products are necessarily the best available for the purpose. 


\section{INTRODUCTION}

Historically, mechanical assembly was predicted to be the dominant application domain of industrial robots; yet assembly tasks are still primarily addressed by manual labor. New advances in force control (FC), machine vision, and robot dexterity are enabling industrial robots, such as four to seven degree of freedom (DOF) arms and selective compliant assembly robot arm (SCARA) manipulators, to catch up with the original visions of robotic assembly. As these technologies progress, performance metrics and associated test methodologies are needed to unify research efforts, characterize the state of technology, and to provide a means for end-users to evaluate the capabilities of robotic assembly systems.

This report describes the current state of force-controlled mechanical assembly, and highlights key robot technologies and algorithms that enable this utilization. Section II gives an overview of the use of robots for assembly tasks. Section III provides insight into the role of dexterity in assembly robotics. Section IV discusses various strategies used by automated systems to enable assembly. Section V describes frequently-used robot force control algorithms. Section VI provides an overview of force control options currently commercially available from robot manufacturers. Section VII discusses the metrics used to assess both force control stability and assembly efficacy. Section VIII introduces some concepts for performance testing utilizing the defined metrics.

\section{ROBOTICS IN ASSEMBLY}

Within the domain of industrial automation, the roles of robots have been relegated largely to minimal-contact, repetitive, position-controlled tasks such as spot welding, spray painting, and the transportation, packaging, and handling of materials. Such applications use the known physical properties and feedback properties of the robot to localize the robot as it moves through the Cartesian space. Robots commanded by position control algorithms can be programmed to perform their tasks independently with little knowledge of the environment in which they operate. However, strict position-controlled robots are incapable of performing high tolerance assemblies due to the cumulative uncertainties of assembly parts, links, axles, fixtures, and end effectors that exceed component clearances. Unable to overcome these shortcomings, position-controlled robots achieve assemblies through the use of chamfers, external feedback mechanisms (e.g., ultrasound or light range), and position compensation tools such as remote center of compliance (RCC) devices that passively accommodate forces and torques to allow the parts being held to adjust to minor lateral and angular uncertainties. Although these tools allow for the assembly of components, they are not general-purpose solutions, and the product features which allow them to compensate for cumulative uncertainties are not conducive to current trends in shrinking component designs.

Compliant motion control is a robot control method that modulates robot position and velocity based on measured contact forces [1]. Using this sense of touch, robots can perform tasks requiring physical contact between the end effector or workpiece and the manufacturing process. Despite these advances, however, due to bandwidth limitations and unreliable system performance [2] industry has been a slow adopter, and processes requiring extended contact between the manipulator and assembly components have largely remained the task domain of manual labor forces. 
Though force control has been an active field of research for decades, the industrial world has been slow to adopt force-based practices in automating assembly tasks [3]. There are several factors that have influenced this slow migration, not the least of which is the cost involved, both in terms of the time necessary to implement and the steep learning curve. Some of the most prohibitive aspects are the failed promises of reliability and throughput; reliable solutions are not fast, and fast solutions are not reliable. While a robot can be programmed to be an expert at a single assembly problem, generalizing this expertise across all assemblies is difficult. Some of these problems are being addressed with new concepts of collaborative robotics where a human compensates for a robot's inadequacies to complete an assembly operation. The safety implications of robot operation within such a close proximity to a human presents additional control requirements $[4,5]$.

Emerging research topics in task-driven human-robot collaboration have focused on the direct interaction between a human operator and an industrial manipulator. Robots intended to work with humans often incorporate force-sensing technologies and control algorithms to enable human-guided training [6], object handling [7, 8], and safe collision handling [9].

\section{ASSEMBLY AND ROBOT DEXTERITY}

A limiting factor in the implementation of robot assembly cells is the dexterity of the manipulator and end effector. Though the utility of a standard 6DOF robot arm with a traditional gripper has been demonstrated through decades of implementation, the actual number of tasks capable of being performed by such configurations is limited. Assembly configurations must be designed with the limitations of the robot in mind. Such restricting factors include the robot's reach, lift capacity, sensing capabilities, and dexterity.

It is generally agreed that increasing the dexterous capabilities of the robot or the gripper will enable the robot to overcome task obstacles by being more flexible [10]. However, there is relatively little consensus regarding the form and measurable degree of such dexterity $[10,11]$. For example, increasing system dexterity may be accomplished by either adding additional linkages in the arm kinematics or by attaching a gripper featuring withinhand dexterity (such as anthropomorphic or multi-fingered hands with numerous independently-controllable degrees of freedom). Both extend the repertoire of abilities of the robot, but each in its own distinct way and each adding complexity in the control domain. As an example, robots with redundant kinematics are more nimble and capable of navigating obstruction-laden work zones, whereas dexterous grippers enable reconfigurable grasps and flexible manipulation of work objects.

Gripper dexterity, in particular, has been the topic of considerable research within the past few years. Though most investigations have focused on grasping (e.g., algorithms, modeling, performance, and optimization [12-16]), investigations into within-hand manipulation of parts have the potential for positively impacting a robot's capabilities for assembly. Capabilities such as regrasping (releasing and reacquiring objects to change their position and orientation), finger gaiting (alternating contact between fingers in order to stabilize or regrasp parts, e.g., [17]), finger pivoting (where two fingers grasping an object form an axis of rotation, and a third finger rotates the object along that axis, e.g., [18]), stable in-grasp manipulation of parts for repositioning and reorienting [19], and unstable ingrasp manipulation [20, 21]. 


\section{Assembly Strategies}

Many common implementations of force control for assembly implement force-driven motion primitives such as push, pull, and twist to join two or more parts together. Such approaches require controlled fixturing to ensure some level of position and process certainty. Further, they are reminiscent of the classic position-controlled approaches of robotics in that the manipulator is simply performing a set of basic motions on parts with little in-line process validation, and they do not reliably handle part variance.

To address the shortcomings of the motion primitive approach, adaptive techniques in the form of event-based search strategies recognize and attempt to compensate for part and position variance. In many cases a given assembly task may have numerous strategies defined for its completion. For instance, a peg-in-hole assembly may be completed by "dumb" searches in which the peg to be inserted is moved around a candidate hole position (either randomly, or by a structured geometric pattern like rasters [12] or spirals [23]) until it can be pushed in. Alternatively, more explicit methods can be employed that intelligently probe the candidate hole position and, based on the effector position and force moments, accurately identify the location and orientation of the hole into which the peg will be inserted [24]. Other assemblies that are more specific in nature (for example, automobile clutch assemblies) can be parameterized and generalized [22], or broken down into separate motion primitives [23].

Further, most mechanical assemblies employ chamfers to eliminate sharp edges that lead to stress concentration. These may also be used to provide tactile clues for parts guidance and permit the use of passive tools like RCCs [25], though it has been demonstrated that insertion assemblies can be performed in their absence [26]. Other efforts to address the assembly problem have focused on modeling tasks for assessment and optimization. Such models are typically abstract in nature, and are used to describe solutions for design and scheduling problems through relational representations [27, 28], component connections [29], assembly “intent” [30], and cumulative entropies [31].

\section{FORCE CONTROL}

Tasks that require a robotic manipulator to directly interact with its environment are dependent on the robot's ability to sense the world it touches. Merely sensing forces and torques, however, is insufficient for understanding them. These sensed forces must be put into context of the robot's current configuration, and must subsequently result in a marked and appropriate change in behavior. Compliant motion control (or force control, or torque control) is a reactive control model in which a tight control loop is expected to make comparatively simple decisions based on the measured signal outputs of a variety of sensors measuring velocity, position, acceleration, and force. These four variables comprise the basic components of a myriad of force control methods.

The application of force control is essentially one of relating measured forces to one or more system variables in the form of a mass-spring damper for tuning and stability. There exists a wide variety of compliant motion control algorithms relating measured force to virtually any combination of position, velocity, acceleration, and applied force [32-34]. The remainder of this section consists of a discussion of the principal force control algorithm types: explicit force control, stiffness control, impedance control, admittance (or accommodation) control, implicit force control, and natural admittance control. 


\section{A. Explicit Force Control}

The most basic active force control algorithm is that of explicit force control, in which the measured forces are related to the desired, applied forces [35]. The sensed forces, $F_{S}$, are used in a tight feedback loop to generate force errors, which are then used directly to adjust the commanded force variables, $F_{C}$. In schematic form, this can be illustrated as is shown in Figure 1.

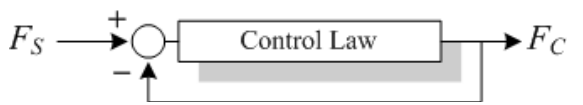

Figure 1: Generalized explicit force control. The differences between the measured and commanded force values drive the robot's motions.

The force control law is typically simple in implementation, such as the proportionalintegral (PI) control illustrated in Figure 2, where the error between the measured and commanded/sensed forces, $e$, results in more or less force being applied over time, $t$. The rate of convergence is controlled by the $\mathrm{P}$ and I gain values, $K_{P}$ and $K_{I}$ respectively.

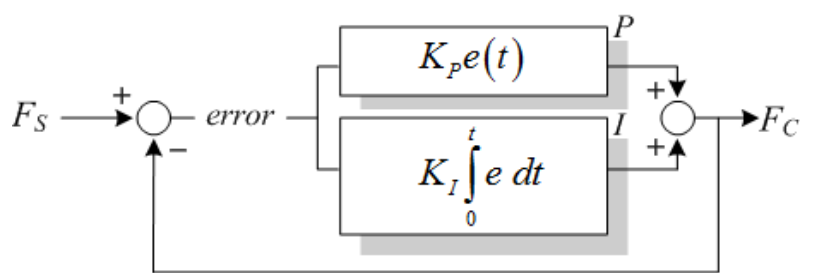

Figure 2: Explicit force control with PI control. Proportional and integral control values, $\mathrm{P}$ and $\mathrm{I}$, drive the convergence between measured and commanded force values, $F_{S}$ and $F_{C}$.

\section{B. Stiffness Control}

One of the most basic and fundamental variants of force control for robotics, stiffness control, softens the impacts and incurred forces of the robot's end effector by mimicking the properties of springs. The trivial implementation of this is to literally utilize mechanical constructs composed of springs and dampers inline with the robot effector. So-called passive stiffness control mechanisms are well-established and understood, with tools being used to compensate for minor errors in orientation for insertion tasks [36]. In contrast, active stiffness control effectively turns the robot into a programmable spring, with the stiffness of the robot controlled by means of force feedback in the closed-loop system. Stiffness control is most appropriate for low-bandwidth applications that focus on accuracy in force measurements (e.g., [37]).

\section{Impedance Control}

Impedance control measures the user's motion input, and then feeds back a reaction force to the operator [38]. Essentially, the output force is driven by the operator's commanded velocity. The relationship between the input velocity and the applied force is classically referred to as the mechanical impedance of the robot. Impedance control attempts to simplify the control paradigm by maintaining the dynamic relationships between robot variables such as tool center point (TCP) position and applied force, rather than maintaining the individual variables themselves (as is typical with most force-based robot control algorithms). Providing high bandwidth force responses for stable tool contact with a rigid object, impedance control is typically used for high-speed applications such as polishing, metal removal, and certain assembly tasks. 


\section{Admittance / Accommodation Control}

The opposite of impedance control, admittance (or accommodation) control [25] measures the forces exerted by the user, and then feeds them back in the form of velocity. In short, the velocity of the robot is a function of the commanded force. The stiffness and dampening properties of the system can be adjusted to increase or decrease the velocity of the robot end effector based on the applied/commanded forces.

\section{E. Implicit Force Control}

In contrast with the explicit force control loop discussed earlier, there is no actual force feedback into the system with implicit force control. Instead, the forces exerted by the robot's end effector are implied based on a priori knowledge of applied torques given measured joint currents. In one such implementation, a variation of passive stiffness control, a robot outfitted with elastic joints has its TCP moved to pre-defined positions on the surface of an object (e.g., [39]). The resulting applied forces at those positions are inferred based on a priori knowledge obtained from earlier measurements. Such methods however, are impractical given part variations and encoder drift. A more common instantiation, however, infers applied forces based on either applied torques or measured currents at each joint of the manipulator (e.g., [40]). In both cases, the applied forces are measured and calibrated using an external force transducer.

\section{F. Natural Admittance Control}

Another algorithm designed to imitate the properties of mechanical systems, natural admittance control implements virtual springs and dampers to control the robot's dynamics as it is drawn toward a controllable virtual attractor [41]. The attractor interacts with a reference point, fixed relative to the robot's tool frame; virtual impedances and physical exerted forces interact to effect motion. The robot equilibrates virtual and physical forces by accelerating in the direction of the net force, scaled by the inverse of the robot's inertia, producing both stable and responsive interactions with the robot effector.

\section{COMMERCial RoBot SOLUTIONS}

This section summarizes the commercially available robot force control and assembly options available at the time this report was written. Table 1 provides a brief summary of the robot manufacturers and their force control solutions based on a review of available product manuals, brochures, and specifications.

The information provided in Table 1 is intended to establish a brief overview of the forcebased control options available from various industrial robot manufacturers. For each manufacturer we indicate whether the control algorithms and force/torque sensors are provided either as a function that comes standard with the purchase of a robot or as an elective purchase option. Similarly, we specify which force control methods are provided by the manufacturer, as well as which motion primitives are enabled while force control is active. Though all of the manufacturers in Table 1 offer some form of force control, relatively few offer a force-based assembly option. Those that do have an assembly option are indicated, as well as whether or not they come standard with the force control option. 
Table 1: Overview of Robot Manufacturers Who Offer Force-Based Control Options

\begin{tabular}{|c|c|c|c|c|c|}
\hline & Adept & ABB & Barrett & DENSO & EPSON \\
\hline $\begin{array}{c}\text { FC Purchase } \\
\text { Option (Control) }\end{array}$ & $\begin{array}{l}\text { Standard (guard/ } \\
\text { protection } \\
\text { function) }\end{array}$ & Option & Standard & Standard & $\begin{array}{l}\text { Option (guard/ } \\
\text { protection } \\
\text { function) }\end{array}$ \\
\hline $\begin{array}{l}\text { FC Purchase } \\
\text { Option (Sensor) }\end{array}$ & Option & $3^{\text {rd }}$ party option & Standard/option & None & $\begin{array}{l}\text { Standard (joint } \\
\text { torques)/option } \\
\text { (force) }\end{array}$ \\
\hline $\begin{array}{c}\text { Native Force } \\
\text { Control Method } \\
\end{array}$ & None & $\begin{array}{c}\text { Accommodation } \\
\text { control }\end{array}$ & $\begin{array}{c}\text { Implicit force } \\
\text { control }\end{array}$ & $\begin{array}{c}\text { Per-axis implicit } \\
\text { force control }\end{array}$ & None \\
\hline $\begin{array}{l}\text { FC Assembly } \\
\text { Option }\end{array}$ & None & $\begin{array}{c}\text { Native with FC } \\
\text { option }\end{array}$ & None & None & None \\
\hline $\begin{array}{c}\text { Force-Based } \\
\text { Motion } \\
\text { Primitives }\end{array}$ & None & $\begin{array}{l}\text { Constant push, } \\
\text { search primitives }\end{array}$ & Cartesian motions & None & None \\
\hline $\begin{array}{l}\text { FC-Enabled } \\
\text { Robot Models }\end{array}$ & $\begin{array}{l}\text { 4/6DOF arms, } \\
\text { SCARA }\end{array}$ & $\begin{array}{c}\text { 6DOF arms, } \\
\text { 14DOF prototype }\end{array}$ & 4/7DOF arms & 4/6DOF arms & $\begin{array}{l}\text { 4/6DOF arms, } \\
\text { SCARA }\end{array}$ \\
\hline \multirow[t]{2}{*}{$\begin{array}{c}\text { FC Sensor } \\
\text { Location }\end{array}$} & TCP & ТCP & Joints, TCP & Joints & TCP \\
\hline & FANUC & KUKA & $\begin{array}{c}\text { Motoman- } \\
\text { Yaskawa }\end{array}$ & RRC & Stäubli \\
\hline $\begin{array}{c}\text { FC Purchase } \\
\text { Option (Control) }\end{array}$ & Option & $\begin{array}{l}\text { Standard (7DOF } \\
\text { arm)/option }\end{array}$ & Option & Standard & Option \\
\hline $\begin{array}{c}\text { FC Purchase } \\
\text { Option (Sensor) }\end{array}$ & Option & $\begin{array}{l}\text { Standard (7DOF } \\
\text { arm)/ } 3^{\text {rd }} \text { party } \\
\text { option }\end{array}$ & $3^{\text {rd }}$ party option & Standard & $3^{\text {rd }}$ party option \\
\hline $\begin{array}{l}\text { Native Force } \\
\text { Control Method }\end{array}$ & $\begin{array}{l}\text { Impedance } \\
\text { control }\end{array}$ & $\begin{array}{l}\text { Explicit force } \\
\text { control, } \\
\text { impedance } \\
\text { control (7DOF } \\
\text { arm) }\end{array}$ & $\begin{array}{l}\text { Explicit force } \\
\text { control }\end{array}$ & $\begin{array}{c}\text { Impedance } \\
\text { control, stiffness } \\
\text { control }\end{array}$ & $\begin{array}{l}\text { Accommodation } \\
\text { control }\end{array}$ \\
\hline $\begin{array}{l}\text { FC Assembly } \\
\text { Option }\end{array}$ & $\begin{array}{l}\text { Native with FC } \\
\text { option }\end{array}$ & $\begin{array}{l}\text { None (option in } \\
\text { pipeline) }\end{array}$ & None & None & None \\
\hline $\begin{array}{l}\text { Force-Based } \\
\text { Motion } \\
\text { Primitives }\end{array}$ & $\begin{array}{l}\text { Constant push, } \\
\text { search \& insert } \\
\text { primitives }\end{array}$ & $\begin{array}{l}\text { Constant push, } \\
\text { force-based linear } \\
\text { and joint motions }\end{array}$ & Constant push & $\begin{array}{c}\text { Force-based } \\
\text { linear and joint } \\
\text { motions }\end{array}$ & $\begin{array}{c}\text { Force-based } \\
\text { linear and joint } \\
\text { motions }\end{array}$ \\
\hline $\begin{array}{c}\text { FC-Enabled } \\
\text { Robot Models }\end{array}$ & $\begin{array}{c}\text { 6DOF arms } \\
\text { (limited models) }\end{array}$ & 6/7DOF arms & 6/7DOF arms & 7DOF arms & $\begin{array}{l}\text { 6DOF arms, } \\
\text { SCARA }\end{array}$ \\
\hline $\begin{array}{l}\text { FC Sensor } \\
\text { Location }\end{array}$ & ТCP & $\begin{array}{c}\text { Joints (7DOF } \\
\text { arm), TCP }\end{array}$ & TCP & Joints & ТCP \\
\hline
\end{tabular}

\section{METRICS FOR ASSESSING FORCE CONTROL}

A notable shortcoming of the assessment of force control efficacy is the fundamental lack of comprehensive (and widely-accepted) metrics for evaluation. Such evaluations are difficult to quantify as they are generally specific to the respective application, and may further be arbitrary according to the user's preferences. Within the domain of force control, however, certain qualifiable trends in assessment do repeat. For all such assessments, it is presumed that performance evaluations are measured by competent users utilizing traceable procedures and equipment as defined by the applicable metrological fields.

\section{A. Force Control Metrics}

As was discussed earlier, force control algorithms are based on common feedback loops with tunable dampers, springs, and associated gains. The algorithms provide a means of physically interacting with the world while limiting the potential for damaging either the objects within it or to the robot itself. This goal cannot be achieved if the robot's actions are not tightly controlled. As such, one of the largest overlying themes of robot performance evaluation is that of stability. A properly damped system, when 
encountering variances in incidental force measurements, will react to said forces and eventually settle to a stable (sustainable) configuration until new changes in force measurements are encountered. An improperly damped system, however, will resonate, with a worst-case scenario resulting in the magnitudes of the oscillations increasing until a catastrophic event occurs.

Settle Stability: How long does the system take to settle? A common test of this is for the robot tool tip to move toward a stable surface until initial impact, and then record the length of time necessary for the robot to settle such that it maintains a consistent contact force with a surface, a limit cycle with a constant frequency and magnitude. A stable system will settle fairly quickly - typically after only one or two consecutive "hops" (brief moments of motion reversal along an approach vector) of decreasing magnitudewhereas an unstable system will hop indefinitely with varying (or, worse, increasing) magnitudes.

Obstruction Stability: If an immovable object keeps the robot tool from reaching a goal state, does the robot maintain stable, constant contact, or does the incurred force increase? A stable system will impact, hop slightly due to motion reversal, and then come to rest against the object quickly. If there is some perceptible slope to the incident force, the robot may move along the lowest-resistant route. This permits the robot effector to trace up a slope or around an object in an effort to complete its program.

Control Switch Stability: More of a metric for assessing the capabilities of the robot controller, determining the stability of a robot when switching between control structures (such transitioning from position control to impedance control) can prove indicative of the system's handling of force and position errors. A stable system will transition from one control structure to another smoothly and without incident, while an unstable system may jerk when transitioning due to unequilibrated offset and encoder errors or lapses in time response for poorly-tuned control loops.

Surface Cohesion (Force Profiles): While moving along non-planar surfaces, a robot is often required to maintain constant surface contact for a given task such as paint removal, welding, and deburring. For some applications, the force with which it maintains contact is as important as the maintenance of contact. Stable systems will respond quickly to changes in the surface and maintain a relatively constant applied force, while less responsive systems may skip or gouge the surface. Similarly, the speed at which the robot moves along a surface and maintains force stability is also a factor in assessment for relevant applications.

Incurred Force Limitations: As the robot performs motion tasks, it is likely to incur a number of incident forces while interacting with objects. For safety and quality assurance purposes, it is important that these incurred forces do not exceed defined limits. Safe responses to these forces include reacting to the forces along a vector in the direction of the applied force, slowing/braking the robot, or halting the robot altogether.

\section{B. Force-Based Assembly Metrics}

Numerous automation tasks and process controls can be addressed by means of relatively simple damped system solutions such as proportional-integral-derivative (PID) or massspring dampers. However, complex tasks such as multi-staged assemblies with component location uncertainties require an adaptive system capable of automatically 
adjusting to both identify and compensate for changes in operational conditions. This capacity may either be explicitly programmed or automatically adapted to via machine learning. In either case, some form of feedback basis is required for the optimization process; this task-specific feedback (e.g., time or bandwidth) is considered for process optimization, and is distinct from the feedback for force-control parameter tuning. In this section, we briefly describe a number of metrics used for assembly optimization.

Assembly Time: Efforts in automating mechanical assemblies is for naught if the resulting product cannot be completed quickly. As such, a common metric for automation efficacy is the required time to complete an assembly task [42, 43].

Success Rate: As important as the time required to complete an assembly, successfully completing assemblies rather than prematurely aborting them due to time constraints or improperly seated parts is an integral component of process automation. For this reason, the number of successful assemblies [44] is often seen as a useful metric for system throughput and stability.

Incurred (Maximum/Average) Force: An assembly task requires part-on-part contact, and as such runs the risk of parts damage. A trivial solution to an assembly attempt is to merely apply more force, which, while potentially completing the assembly faster, also risks the structural integrity of the parts, the robot, the tooling, and the force sensors, themselves. As such, one would prefer to minimize the forces either incurred or encountered to minimize damage potential [42]. Unfortunately, there is no "ideal" value for an incurred assembly force. Instead, the material properties of the components being assembled will often dictate the softness of touch for handling. Small and fragile parts require gentle touch, whereas more sturdy materials can withstand more punishment. Ideally, assembly forces are controlled to be minimally sufficient. A notable subset of the incurred force metric is the measurement of impact forces. Often three to five times larger than the forces incurred throughout the process of completing an assembly, force spikes resulting from the initial impact between the robot tool and parts being assembled are used as indicators of the robot's ability to sense and quickly react to forces [45, 46]. Well-tuned algorithms and systems react quickly, mitigating the impact force.

\section{VIII.ASSESSING THE PERFORMANCE OF ROBOTIC ASSEMBLY}

This section presents the concept of an independent measurement system developed by the National Institute of Standards and Technology for assessing the performance of a robotic assembly against the proposed metrics found in section VII. Figure 3 shows a prototype design for an independent measurement system. The design incorporates a 6-axis load cell fixtured between two base plates. The bottom base plate supports the overall system while the top base plate is designed to accept a set of modular artifacts. Each artifact is designed to address one or more control or assembly metrics.

While it is possible to access force data directly from a robotic system's force sensing system and derive the defined metrics, these measurements would be based on the inherent properties of the system under test. An independent measurement system allows for comparative metrics between systems without effects such as force accuracies, data latencies, and the effects of tooling offsets. 


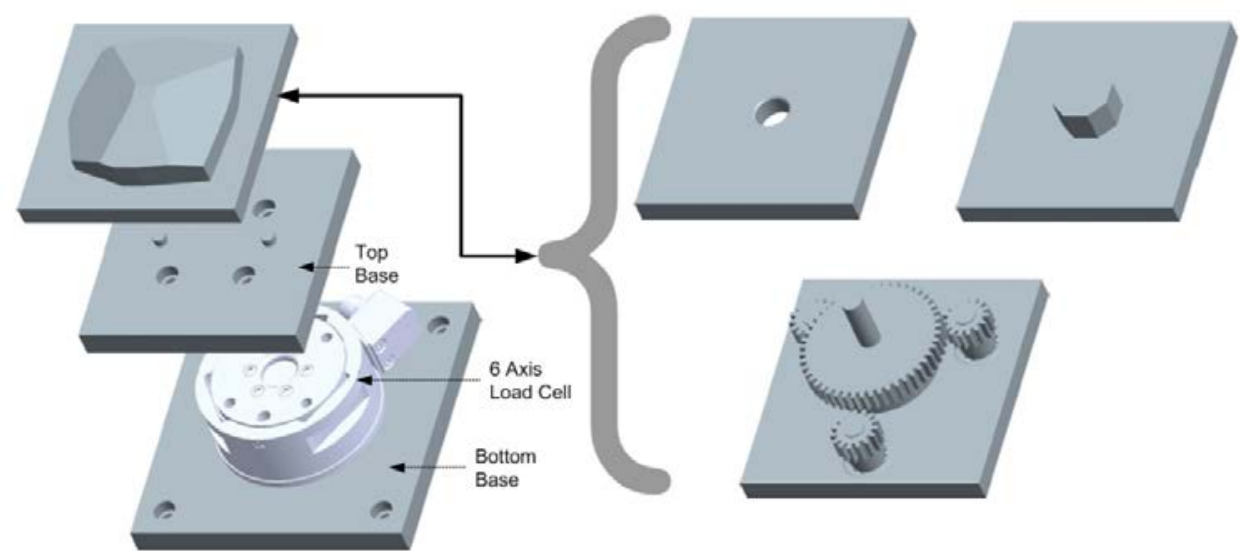

Figure 3: Independent measurement system for evaluation of force control and force-based assembly metrics

Figure 4 shows an artifact for measuring obstruction stability on a force controlled robotic system capable of adapting tool paths in the presence of an unanticipated slope. The artifact contains several degrees of slope from slight to aggressive at various artifact locations to support several tool path directions and eliminate the need to reorient the artifact relative to the robot coordinate frame.
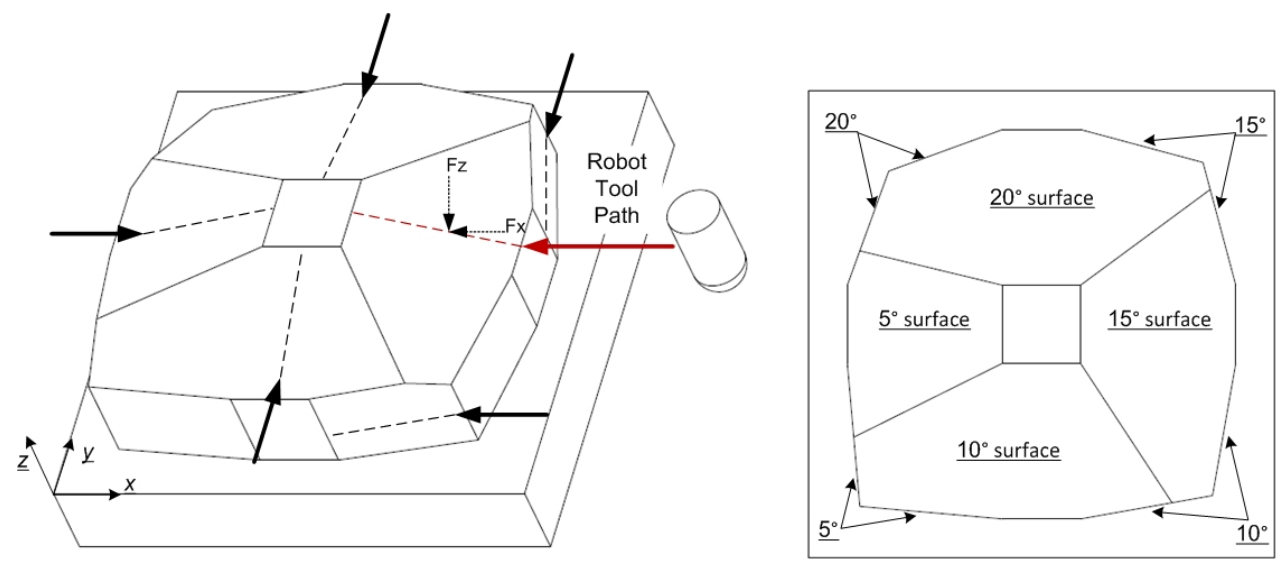

Figure 4: Test artifact for obstruction stability with provisions for obstruction avoidance

Figure 5 contains an artifact for measuring the settle stability and obstruction avoidance capabilities of a system and another artifact for measuring the control switch stability of a force based robotic assembly system. Settling stability is measured as the robot tooling collides with each surface of the artifact when robot tool paths are programmed to move toward the load cell center along the primary axes and at $45^{\circ} \mathrm{XY}$ planar trajectories. In the case of control switch stability, the robot tooling is inserted into the hole designed to allow slip but not any XY movement until the tool just touches the hole bottom in the $\mathrm{Z}$ direction. A control switch is performed and the reactive forces are measured. 


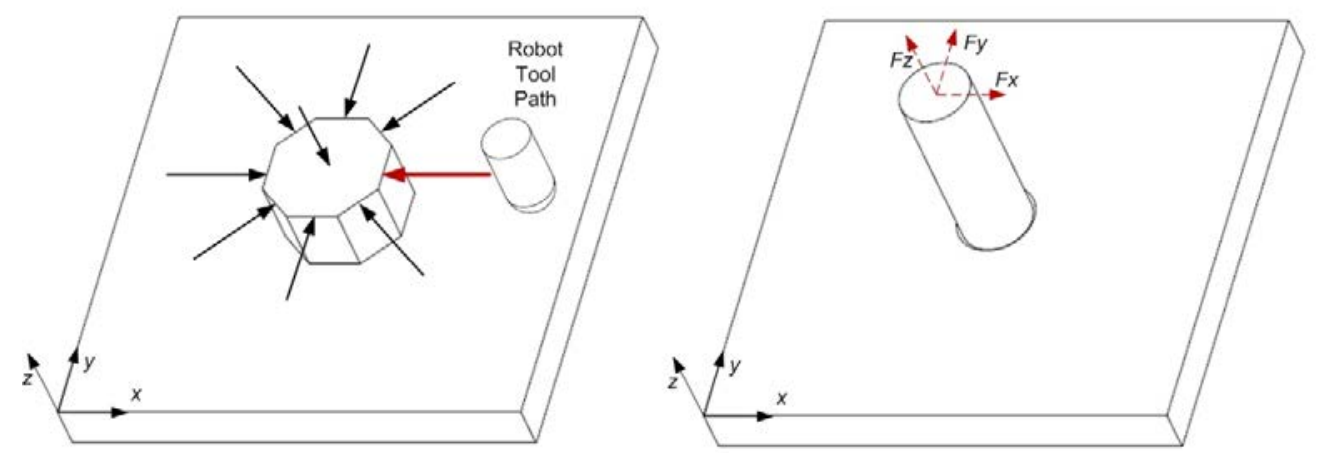

Figure 5: Test artifact for settle stability and obstruction avoidance (left) and control switch stability given a peg inserted into a hole (right)

Figure 6 contains a proposed artifact for measuring the forces associated with a hole searching algorithm and another artifact for measuring the forces associated with the insertion of a sun gear into a planetary gear system. These component assembly operations are designed such that the center of the assembled component resides at the center of the independent load cell.

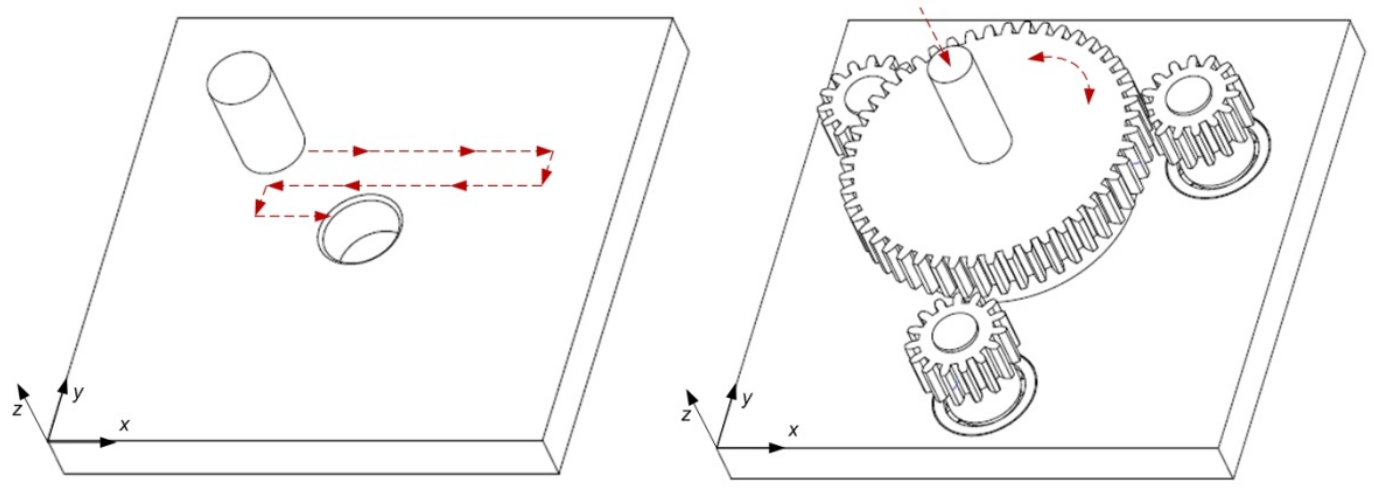

Figure 6: Test artifact for force based assembly metrics for hole searching (left) and planetary gear insertion (right)

The artifacts presented above represent a subset of artifacts that will be developed for this independent measurement system for evaluating force-based robotic assembly. This measurement system along with the defined performance metrics will help robot suppliers better specify robot capabilities. The system will also provide a mechanism for the research community when developing new force-based control algorithms for assembly. Finally, the measurement system will provide end users with a mechanism for assessing robotic assembly system capabilities prior to limited production testing. As these measurement system concepts progress, multiple component assembly operations may be designed into assembly kits. An assembly kit is a collection of components classed by specific manufacturing sectors that can be used to assess the overall performance of a robotic assembly system. In addition, test methods will be derived that will include measures for assessing dexterity in conjunction with assembly processes. 


\section{REFERENCES}

[1] J. De Schutter. "A Study of Active Compliant Motion Control Methods for Rigid Manipulators Based on a Generic Scheme." Proceedings of the IEEE International Conference on Robotics and Automation. 1987. Pp. 1060-1065.

[2] S. Eppinger, and W. Seering. "Understanding Bandwidth Limitations in Robot Force Control.” Proceedings of the IEEE International Conference on Robotics and Automation. 1987. Pp. 904-909.

[3] D. Gravel, F. Maslar, G. Zhang, et al. "Toward Robotizing Powertrain Assembly." Proceedings of the $7^{\text {th }}$ World Congress on Intelligent Control and Automation. 2008. Pp. 541-546.

[4] S. Haddadin, S. Haddadin, A. Khoury, et.al. "On Making Robots Understand Safety I: Embedding Injury Knowledge into Control.” International Journal of Robotic Research. 2012. Accepted.

[5] S. Oberer, A. Puzik, \& A. Verl, "Measuring the Collision Potential of Industrial Robots", Proceedings of the $41^{\text {st }}$ ISR and $6^{\text {th }}$ ROBOTIK. 2010. Pp. 98-101.

[6] W. Newman, C. Birkhimer, \& R. Hebbar. "Towards Automatic Transfer of Human Skills for Robotic Assembly." Proceedings of the IEEE/RSJ International Conference on Intelligent Robots and Systems. 2003. Pp. 2528-2533.

[7] K. Kosuge, S. Hashimoto, \& H. Yoshida. "Human-Robots Collaboration System for Flexible Object Handling.” Proceedings of the IEEE International Conference on Robotics and Automation. 1998. Pp. 1841-1846.

[8] T. Takubo, H. Arai, \& K. Tanie. "Human-Robot Cooperative Handling Using Virtual Nonholonomic Constraint in 3-D Space.” Proceedings of the IEEE International Conference on Robotics and Automation. 2001. Pp. 2680-2685.

[9] S. Haddadin, M. Suppa, S. Fuchs, et al. "Towards the Robotic Co-Worker." Springer Tracts in Advanced Robotics. 2011. 70:2011. Pp. 261-282.

[10] R.R. Ma \& A.M. Dollar. "On Dexterity and Dexterous Manipulation." Proceedings of the 15th International Conference on Advanced Robotics. 2011. Pp. 1-7.

[11] A. Bicchi. "Hands for Dexterous Manipulation and Robust Grasping." IEEE Transactions on Robotics and Automation. 2000. 16:6. Pp. 652-662.

[12] Y. Li, J. Keesling, J. English, et al. "Design, Creation, and Validation of a Comprehensive Database Infrastructure for Robotic Grasping." Proceedings of the IEEE/RAS-EMBS International Conference on Biomedical Robotics and Biomechatronics. 2008. Pp. 335341.

[13] J. Bohg \& D. Kragic. "Learning Grasping Points with Shape Context." Robotics and Autonomous Systems. 2010. 58:4. Pp. 362-377.

[14] N. Jetchev \& M. Toussaint. "Trajectory Prediction in Cluttered Voxel Environments." Proceedings of the IEEE International Conference on Robotics and Automation. 2010. Pp. 2523-2528.

[15] J.A. Jorgensen \& H.G. Petersen. "Usage of Simulations to Plan Stable Grasping of Unknown Objects with a 3-Fingered Schunk Hand." Proceedings of the 41st International Symposium on Robotics. 2010. Pp. 1-6.

[16] Y. Bekiroglu, K. Heubner \& D. Kragic. "Integrating Grasp Planning with Online Stability Assessment Using Tactile Sensing." Proceedings of the IEEE International Conference on Robotics and Automation. 2011. Pp. 4750-4755.

[17] L. Han \& J.C. Trinkle. "Dextrous Manipulation by Rolling and Finger Gaiting." Proceedings of the IEEE International Conference on Robotics and Automation. 1998. Pp. 730-735 
[18] D. Rus. "Dexterous Rotations of Polyhedral." Proceedings of the IEEE International Conference on Robotics and Automation. 1992. Pp. 2758-2763.

[19] D. Osswald \& H. Worn. "Mechanical System and Control System of a Dexterous Robot Hand." Proceedings of the IEEE/RAS International Conference on Humanoid Robots. (2001).

[20] A. Bicchi \& R. Sorrentino. "Dexterous Manipulation Through Rolling." Proceedings of the IEEE International Conference on Robotics and Automation. 1992. Pp. 452-457.

[21] D.L. Brock. "Enhancing the Dexterity of a Robot Hand Using Controlled Slip." Proceedings of the IEEE International Conference on Robotics and Automation. 1988. Pp. 249-251.

[22] "Force Sensor Operator's Manual." FANUC Robot Series. MARA3I2L107901E Rev. C. Rochester Hills, MI, USA.

[23] "Robotics Application Manual: Force Control for Assembly." ABB Automation Technologies BA, Robotics. SE-721 68. Vasteras, Sweden.

[24] W.S. Newman, Y. Zhao \& Y. Pao. "Interpretation of Force and Moment Signals for Compliant Peg-in-Hole Assembly." Proceedings of the IEEE International Conference on Robotics and Automation. 2001. Pp. 571-576.

[25] D.E. Whitney. "Force Feedback Control of Manipulator Fine Motions." ASME Journal of Dynamic Systems, Measurement and Control. 1977. 99. Pp. 91-97.

[26] M.E. Caine, T. Lozano-Perez \& W.P. Seering. "Assembly Strategies for Chamferless Parts." Proceedings of the IEEE International Conference on Robotics and Automation. 1989. Pp. 472-477.

[27] D. Deneux. "Introduction to Assembly Features: An Illustrated Synthesis Methodology." Journal of Intelligent Manufacturing. 1999. 10. Pp. 29-39.

[28] A. Gayretli \& H.S. Abdalla. "A Feature-Based Prototype System for the Evaluation and Optimization of Manufacturing Processes." Computers and Industrial Engineering. 1999. 37. Pp. 481-484.

[29] W. Van Holland \& W.F. Bronsvoort. "Assembly Features in Modeling and Planning." Robotics and Computer Integrating Manufacturing. 2000. 16. Pp. 277-294.

[30] H. Ullah, E.L.J. Bohez \& M.A. Irfan. "Assembly Features: Definition Classification, and Instantiation." Proceedings of the IEEE International Conference on Emerging Technologies. 2006. Pp. 617-623.

[31] A.C. Sanderson. "Parts Entropy Methods for Robotic Assembly System Design." Proceedings of the IEEE International Conference on Robotics and Automation. 1984. Pp. 600-608.

[32] D.E. Whitney. "Historical Perspective and State of the Art in Robot Force Control." Proceedings of the IEEE International Conference on Robotics and Automation. 1985. Pp. 262-268.

[33] G. Zeng \& A. Hemami. "An Overview of Robot Force Control." Robotica. 1997. 15. Pp. 473-482.

[34] T. Lefebvre, J. Xiao, H. Bruyninckx, et al. "Active Compliant Motion: A Survey." Advanced Robotics. 2005. 19:5. Pp. 479-499.

[35] R. Volpe \& P. Khosla. "An Experimental Evaluation and Comparison of Explicit Force Control Strategies for Robotic Manipulators." Proceedings of the International Conference on Robotics and Automation. 1992. Pp. 1387-1393.

[36] D.E. Whitney. "Quasi-Static Assembly of Compliantly Supported Rigid Parts." Journal of Dynamic Systems, Measurement, and Control. 1982. 104:1. Pp. 65-77.

[37] S. Katsura, Y. Matsumoto, K. Ohnishi. "Analysis and Experimental Validation of Force Bandwidth for Force Control.” IEEE Transactions on Industrial Electronics. 2006. 53:3. Pp. 922-928. 
[38] N. Hogan. “Impedance Control: An Approach to Manipulation.” Proceedings of the American Control Conference. 1984. Pp. 304-313.

[39] F. Lange \& G. Hirzinger. "Learning Force Control with Position Controlled Robots." Proceedings of the IEEE International Conference on Robotics and Automation. 1996. Pp. 2282-2288.

[40] J.W.L. Simpson, C.D. Cook, and Z. Li. "Sensorless Force Estimation for Robots with Friction.” Proceedings of the Australian Conference on Robotics and Automation. 2002. Pp. 94-99.

[41] W.S. Newman. "Stability and Performance Limits of Interaction Controllers." Transactions of the ASME Journal of Dynamic Systems, Measurement, and Control. 1992. 114:4. Pp. 563-570.

[42] J.A. Marvel, W.S. Newman, D.P. Gravel, et al. "Automated Learning for Parameter Optimization of Robotic Assembly Tasks Utilizing Genetic Algorithms." Proceedings of the IEEE International Conference on Robotics and Biomimetics. 2008. Pp. 179-184.

[43] J. Wei \& W.S. Newman. "Improving Robotic Assembly Performance through Autonomous Exploration." Proceedings of the IEEE International Conference on Robotics and Automation. 2002. Pp. 3303-3308.

[44] G. Zhang, A. Bell, H. Zhang, et al. "On-Pendant Robotic Assembly Parameter Optimization." Proceedings of the 7th World Congress on Intelligent Controls and Automation. 2008. Pp. 547-552.

[45] D.M. Morris, R. Hebbar \& W.S. Newman. "Force Guided Assemblies Using a Novel Parallel Manipulator." Proceedings of the IEEE International Conference on Robotics and Automation. 2001. Pp. 325-330.

[46] J. Wei. Intelligent Robotic Learning Using Guided Evolutionary Simulated Annealing. Case Western Reserve University. 2001. 